Pacific Journal of Mathematic 


\section{A NOTE ON POLYNOMIAL AND SEPARABLE GAMES}

\section{David Gale ANd Oliver Gross}

1. Introduction. A two-person zero-sum game $\Gamma$ is called polynomial-like or separable if its payoff function is of the form

$$
M(x, y)=\sum_{i=1}^{n} f_{i}(x) g_{i}(y),
$$

where $x$ and $y$ are elements of any strategy sets $X$ and $Y$. Important special cases of separable games are those in which $X$ and $Y$ are bounded (usually compact) subsets of Euclidean spaces and $M$ is a polynomial in the coordinates of $x$ and $y$. These latter are called polynomial games.

It is a basic and fairly elementary fact concerning separable games [1], that, if optimal strategies exist, then these can always be chosen to be finite mixed strategies. We consider here the inverse question: Given a pair of finite mixed strategies, does there exist a separable (respectively, polynomial) game whose unique optimal strategies are the given pair? In case either $X$ or $Y$ is finite the answer is known to be in the negative. We here show, however, that.

THEOREM 1. If $X$ and $Y$ are metric spaces containing infinitely many points and $\mu$ and $\nu$ are any finite mixed strategies on $X$ and $Y$ respectively, then there is a payoff $M$, bounded continuous and separable on $X \times Y$, such that the associated game has $\mu$ and $\nu$ as unique optimal strategies.

CoRollary. If $X$ is a metric space containing infinitely many points and $\mu$ is any finite mixed strategy on $X$, then there is a skew-symmetric payoff $M$, bounded continuous and separable on $X \times X$ such that the associated symmetric game has $\mu$ as the unique optimal strategy.

For the case of polynomial games we show:

THeorem 2. If $X$ and $Y$ are bounded subsets of Euclidean spaces whose closures contain infinitely many cluster points, then for any finite mixed strategies $\mu$ and $\nu$ there exists a polynomial payoff function $M$ such that the associated game has $\mu$ and $\nu$ as its unique optimal strategies.

(An analogous corollary holds here, also.)

Concerning Theorem 2, we remark that Glicksberg and Gross, [2], Received April 30, 1958. 
have shown that any pair of mixed strategies can be the unique solution of a continuous game on the unit square. For finite mixtures, however, their construction is complicated, involving consideration of four special cases, and the payoff function is not a polynomial, nor even separable. The rather simple construction involved in our proof of Theorem 2 shows that their result still holds under the much stronger requirement that the payoff be a polynomial.

Finally, we credit Dresher, Karlin and Shapley, [1], for their rather exhaustive study of the structure of solutions of separable and polynomial games. However, their results do not include the theorems proved in this note. Indeed, one of the above authors has pointed out that the construction of the next section provides a counter-example to one of the conclusions of a structure theorem in [1], and fortunately (for mathematics) an error in the proof of that part of the theorem ${ }^{1}$ was subsequently uncovered.

2. Polynomial games with prescribed unique solutions. This section contains the proof of Theorem 2. Let $X$ and $Y$ be sets satisfying the hypothesis of the theorem (We pause to note that boundedness of $X$ and $Y$ is required to insure integrability, since polynomials may otherwise be unbounded.). Let $\mu$ be the mixed strategy which assigns the weight $\mu_{i}$ to the point $x_{i}$ of $X, i=1, \cdots, m$, where $\sum \mu_{i}=1$. Similarly, let $\nu$ assign the weight $\nu_{j}$ to the point $y_{j}$ in $Y, j=1, \cdots, n$ where $\sum \nu_{j}=1$.

The set of points $\left\{x_{1}, \cdots, x_{m}\right\}$, the spectrum of $\mu$, will be denoted by $\sigma(\mu)$. Similarly, $\sigma(\nu)$ will denote the spectrum of $\nu$.

We now define the following set of polynomials :

$$
\begin{gathered}
f_{0}(x)=\prod_{x \prime \in \sigma(\mu)}\left|x-x^{\prime}\right|^{2}, \\
f_{i}(x)=\prod_{x^{\prime} \in \sigma(\mu)-\left\{x_{i}\right\}} \frac{\left|x-x^{\prime}\right|^{2}}{\left|x_{i}-x^{\prime}\right|^{2}}, \quad i=1, \cdots, m,
\end{gathered}
$$

where $\left|x-x^{\prime}\right|$ is the usual Euclidean distance from $x$ to $x^{\prime}$.

It is clear that the above functions are polynomials; however, aside from continuity, the only properties of them which we shall use are the following:

$$
f_{0}(x) \geq 0 \text { for all } x \in X \text { and } f_{0}(x)=0
$$

if and only if $x \in \sigma(\mu)$.

$$
f_{i}(x) \geq 0 \text { for all } x \in X \text { and } f_{i}(x)=0
$$

if and only if $x \in \sigma(\mu)-\left\{x_{i}\right\},(i=1, \cdots, m)$.

1 Theorem 6, fourth inequality pp. 175-176 of [1]. 


$$
f_{i}\left(x_{i}\right)=1, i=1, \cdots, m \text {. }
$$

In a precisely analogous manner we define the polynomials $g_{0}$ and $g_{j}, j=1, \cdots, n$, on the set $Y$.

Next, let $\alpha_{0}, \alpha_{1}, \cdots, \alpha_{n}$ be $n+1$ distinct cluster points of $\bar{X}$ (the closure of $X$ ) which do not meet $\sigma(\mu)$ (these exist by hypothesis), and define polynomials $\phi$ and $\phi_{j}, j=0, \cdots, n$ on $X$ via

$$
\begin{aligned}
\phi(x) & =\prod_{k=0}^{n}\left|x-\alpha_{k}\right|^{2}, \\
\phi_{j}(x) & =\prod_{\substack{k \neq j \\
0 \leq k \leq n}}\left|x-\alpha_{k}\right|^{2}, \quad j=0, \cdots, n .
\end{aligned}
$$

The only properties of these functions we shall use are that they are all non-negative, that $\phi$ vanishes only on the $\alpha_{k}$, and that $\phi_{j}$ vanishes only on $\alpha_{k}$ with $k \neq j$.

Finally, let $\beta_{0}, \cdots, \beta_{m}$ be $m+1$ distinct cluster points of $\bar{Y}$ which do not meet $\sigma(\nu)$, and define polynomials $\psi$ and $\psi_{i}$ on $Y$ analogous to the functions $\phi$ and $\phi_{j}$ above.

We now define the desired payoff $M$ by

$$
\begin{aligned}
M(x, y) & =f_{0}(x) \phi(x)\left(g_{0}(y) \phi_{0}(x)+\sum_{j=1}^{n}\left(g_{j}(y)-\nu_{j}\right) \phi_{j}(x)\right) \\
& -g_{0}(y) \psi(y)\left(f_{0}(x) \phi_{0}(y)+\sum_{i=1}^{m}\left(f_{i}(x)-\mu_{i}\right) \psi_{i}(y)\right) \\
& -\left(f_{0}(x) \phi(x)\right)^{2}+\left(g_{0}(y) \psi(y)\right)^{2} .
\end{aligned}
$$

We show first that $\mu$ and $\nu$ are optimal strategies. If we compute $M(x, \nu)$ (in the usual extension), we obtain

$$
M(x, \nu)=-\left(f_{0}(x) \phi(x)\right)^{2} \leq 0 .
$$

To see this, it is sufficient to observe that, according to the properties noted above, $\int g_{j} d \nu=\nu_{j}$ and $g_{0}$ and $\psi$ vanish on $\sigma(\nu)$. Similarly, we obtain

$$
M(\mu, y)=\left(g_{0}(y) \psi(y)\right)^{2} \geq 0 .
$$

Thus $\mu$ and $\nu$ are optimal and 0 is the value of the game.

It follows also from (2) above that if $\mu^{\prime}$ is any optimal strategy for player $I$, then the spectrum of $\mu^{\prime}$ is contained in the zeros of $f_{0} \phi$. Thus any optimal $\mu^{\prime}$ has weight only on the pure strategies $x_{i}$ and $\alpha_{j}$, and similarly any optimal $\nu$ for player $I I$ restricts its weight to $\left\{y_{j}\right\} \cup\left\{\beta_{i}\right\}$.

We now show that $\nu$ is the only optimal strategy for player $I I$. For suppose $\nu^{\prime}$ is optimal. Then, in the expression for $M(x, \nu)$, the 
second and fourth terms in (1) drop out in view of the remark of the preceding paragraph and the payoff becomes

$$
\left.M\left(x, \nu^{\prime}\right)=f_{0}(x) \varphi(x)\left[\varphi_{0}(x) \int g_{0} d \nu^{\prime}+\sum_{j=1}^{n} \varphi_{j}(x) \int g_{j}(y)-\nu_{j}\right) d \nu^{\prime}-f_{0}(x) \varphi(x)\right] .
$$

For $x$ close to $\alpha_{0}$ the expression in brackets above approaches $\varphi_{0}\left(\alpha_{0}\right) \int g_{0} d \nu^{\prime}$, and since $\varphi_{0}\left(\alpha_{0}\right)$ is positive we must have $\int g_{0} d \nu^{\prime}=0$. Otherwise the $x$-player by choosing $x$ sufficiently close to $\alpha_{0}$ could achieve a positive payoff, contradicting the optimality of $\nu^{\prime}$. Next, since $\int g_{0} d \nu^{\prime}=0, \nu^{\prime}$ must concentrate all of its mass on the zeros of $g_{0}$, that is, on the points $y_{j}$. Finally, if $\nu_{k}^{\prime} \neq \nu_{k}$ for some index $k$ then the $x$-player could again achieve a positive payoff by choosing $x$ sufficiently close to $x_{k}$. It follows that $\nu^{\prime}=\nu$ as asserted.

Thus, Theorem 2 is established.

3. Metric space games-construction of payoff. This section is dedicated to the construction of the payoff required for the establishment of Theorem 1 and its corollary, which will be proved in the final section. The construction and method of proof are quite similar to those used in proving Theorem 2 ; however, to preserve continuity of presentation, we shall paraphrase identical details.

Therefore, let $X$ and $Y$ be the respective spaces according to hypothesis, $\mu$ and $\nu$ the respective finite probability measures on them. $\mu$ and $\nu$ will be described with the same notations used previously. Finally, let $\rho$ and $\rho^{\prime}$ denote the associated metrics of $X$ and $Y$ respectively. Then, without further ado, we initiate our construction.

The basis of our construction hinges on the fact that any infinite metric space contains a sequence of disjoint neighborhoods. To see this for $X$, say, there is no loss in generality in assuming that $X$ has a cluster point, for otherwise we are guaranteed a sequence by the discrete topology induced by $\rho$ and the infiniteness of $X$. Therefore, let $x^{*}$ denote a cluster point of $X$. First, choose $\alpha_{1} \neq x^{*}$, and, for $i>1$ choose $\alpha_{i}$ so that $0<\rho\left(x^{*}, \alpha_{i}\right)<\rho\left(x^{*}, \alpha_{i-1}\right) / 2$. Then, as our sequence of neighborhoods, $\left\{N_{\alpha_{i}}\right\}$, we set

$$
N_{\alpha_{i}}=\left\{x \mid \rho\left(x, \alpha_{i}\right)<r_{i}\right\}, \quad i=1,2, \cdots,
$$

where $r_{i}=\rho\left(x^{*}, \alpha_{i}\right) / 3$. It is easy to verify, using the triangle inequality, that these neighborhoods are disjoint.

Therefore, let $\left\{N_{\alpha_{i}}\right\}$ denote a sequence of disjoint neighborhoods contained in $X$ (spheres of radius $r_{i}$ centered at $\alpha_{i}$ ). Define functions $\phi_{j}, j=0, \cdots, n$, as follows : 


$$
\phi_{j}(x)=\left\{\begin{array}{cc}
\frac{r_{i}-\rho\left(x, \alpha_{i}\right)}{r_{i}} & \frac{1}{i} \text { if } x \in N_{\alpha_{i}} \text { for some } i \text { (at most one) and } \\
0 \text { otherwise. } & i \equiv j(\bmod (n+1))
\end{array}\right.
$$

One verifies that $\phi_{j}$ is a bounded continuous function on $X$ into the non-negative reals, and which, moreover, satisfies

$$
\phi_{j}\left(\alpha_{i}\right)=\left\{\begin{array}{l}
\frac{1}{i} \text { if } i \equiv j(\bmod (n+1)) \\
0 \text { otherwise }
\end{array}\right.
$$

Next, let the function $\phi$ be given by

$$
\phi(x)=\prod_{i=1}^{m} \rho\left(x, x_{i}\right)
$$

(where, as previously, $\left\{x_{i}\right\}=\sigma(\mu)$ ). There is no question about continuity here. We note merely that

$$
\phi(x)\left\{\begin{array}{l}
=0 \text { if } x \in \sigma(\mu) \\
>0 \text { otherwise. }
\end{array}\right.
$$

Finally, we define functions $f_{j}, j=0, \cdots, m$, as follows :

$$
f_{0}(x)=\phi(x),
$$

and, for $j \in\{1, \cdots, m\}$, set

$$
f_{j}(x)=\prod_{i \neq j} \frac{\rho\left(x, x_{i}\right)}{\rho\left(x_{j}, x_{i}\right)} .
$$

Here, again, continuity is immediate, and we note merely that

$$
f_{j}\left(x_{i}\right)=\delta_{i j}, \quad i, j=1, \cdots, m,
$$

where $\delta$ is Kronecker's delta. Moreover, to insure boundedness of these functions, if such is not the case, we need only replace $\rho$ by the function $\rho /(1+\rho)$ in the formulas (7) and (10) without affecting subsequent arguments.

The remainder of our construction involves defining certain bounded continuous functions on $Y$ into the non-negative reals. To accomplish this we merely repeat the foregoing construction with the replacements:

$$
\begin{aligned}
& \text { " } X \text { " } \rightarrow \text { " } Y \text { ", } \quad \text { " } m \text { " } \rightarrow \text { " } n \text { ", } \\
& \text { " } \rho " \rightarrow \text { " } \rho \text { ", } \quad \text { " } n \text { " } \rightarrow \text { " } m \text { ", } \\
& \text { " } x " \rightarrow \text { " } y ", \quad \text { " } \quad \text { " }, \rightarrow \text { " } \phi ", \\
& \text { " } \alpha \text { " } \rightarrow \text { " } \beta ", \quad \text { " } f \text { " } \rightarrow \text { " } g \text { ", } \\
& \text { "r" } \rightarrow \text { " } r^{\prime},, \quad \text { " } \mu \text { " } \rightarrow \text { " } \nu " \text {. }
\end{aligned}
$$


In terms of these functions, then, and using the convention $\mu_{0}=$ $\nu_{0}=0$, we define our bounded continuous polynomial-like payoff $M$ as follows :

$$
\begin{aligned}
M(x, y) & =-\psi(y) \sum_{j=0}^{m}\left(f_{j}(x)-\mu_{j}\right) \psi_{j}(y) \\
& +\phi(x) \sum_{j=0}^{n}\left(g_{i}(y)-\nu_{j}\right) \phi_{j}(x) \\
& -\phi(x) \sum_{j=0}^{n} \phi_{j}(x)^{2}+\psi(y) \sum_{j=0}^{m} \psi_{j}(y)^{2},
\end{aligned}
$$

$(x, y) \in X \times Y$. This completes our construction.

4. Verification of solution and proof of uniqueness. To verify that $(\mu, \nu)$ is a solution, we calculate first the expectation $M(\mu, y)$ :

$$
M(\mu, y)=\psi(y) \sum_{j=0}^{m} \psi_{j}(y)^{2} \geq 0,
$$

all $y \in Y$.

To see this, we note that the remaining sums vanish by virtue of (8), (9), and (11), i.e. $\phi$ vanishes on $\sigma(\mu)$ and $\int f_{j} d \mu=\mu_{j}, j=0 . \cdots, m$. Similarly,

$$
M(x, \nu)=-\phi(x) \sum_{j=0}^{n} \phi_{j}(x)^{2} \leq 0, \quad \text { all } x \in X .
$$

Thus, $(\mu, \nu)$ is a solution and 0 is the value of the game.

To show uniqueness for the first player, let $\mu^{\prime}$ denote an optimal strategy for him. From the non-negativity of the functions $\phi, \phi_{j}$ in (12), we see that $\int \phi \phi_{j}^{2} d \mu^{\prime}=0$ for all $j \in\{0, \cdots, n\}$ and hence that $\int \phi \phi_{j} d \mu^{\prime}=0$; for otherwise, by (12), a counter strategy is provided by $\nu$. Thus, if $\mu^{\prime}$ is optimal, we have

$$
M\left(\mu^{\prime}, y\right)=-\psi(y) \sum_{j=0}^{m}\left(\mu-\mu_{j}\right) \psi_{j}(y)+\psi(y) \sum_{j=0}^{m} \psi_{j}(y)^{2},
$$

where we have written $\mu_{j}^{\prime}=\int f_{j} d \mu^{\prime}, j=0, \cdots, m$. Next, suppose $\mu_{0}^{\prime}=$ $\int f_{0} d \mu^{\prime} \neq 0$ (and hence, positive). Choose as possible counters a subsequence of the $\beta^{\prime} \mathrm{s},\left\{\beta_{\alpha_{i}}\right\}$ such that $n_{i} \equiv 0(\bmod (m+1))$. Then, by virtue of the minimizer's counterpart of (6), (13) becomes

$$
\begin{aligned}
M\left(\mu^{\prime}, \beta_{n_{i}}\right) & =-\psi\left(\beta_{n_{i}}\right) \mu_{0}^{\prime} \cdot \frac{1}{n_{i}}+\psi\left(\beta_{n_{i}}\right) \frac{1}{n_{i}^{2}} \\
& =\frac{\psi\left(\beta_{n_{i}}\right)}{n_{i}}\left(-\mu_{0}^{\prime}+\frac{1}{n_{i}}\right), \quad i=1,2, \cdots .
\end{aligned}
$$


Since $\psi$ vanishes only on a finite set and is positive elsewhere, we see that the expression above can be made negative for $i$ sufficiently large. Hence $\mu_{0}^{\prime}=0$, and if follows from (8) and (9) that $\sigma\left(\mu^{\prime}\right) \subseteq \sigma(\mu)$, i.e. any optimal $\mu^{\prime}$ must restrict its spectrum to the set $\left\{x_{1}, \cdots, x_{m}\right\}$. Thus, finally, to establish uniqueness, we need only show that the corresponding weights are equal. Let $\mu_{i}^{\prime}$ denote the weight on $x_{i}$ placed by $\mu^{\prime}$. Substituting in our payoff $M$ we obtain (noting $\mu_{0}^{\prime}=0$ ),

$$
M\left(\mu^{\prime}, y\right)=-\psi(y) \sum_{j=1}^{m}\left(\mu_{j}^{\prime}-\mu_{j}\right) \psi_{j}(y)+\psi(y) \sum_{j=0}^{m} \psi_{j}(y)^{2} .
$$

Now suppose $\mu_{k}^{\prime} \neq \mu_{k}$ for some $k \in\{1, \cdots, m\}$. Then, since

$$
\sum_{j=1}^{m} \mu_{j}^{\prime}=\sum_{j=1}^{m} \mu_{j}=1 \text {, }
$$

we would have some $j=j_{0} \in\{1, \cdots, m\}$ such that $\mu_{j_{0}}^{\prime}>\mu_{j_{0}}$. But, by choosing the subsequence $\left\{\beta_{n_{i}}\right\}$ with $n_{i} \equiv j_{0}(\bmod (m+1))$, by the identical argument used before, we would find a counter rendering the expectation (15) negative. Hence $\mu_{j}^{\prime}=\mu_{j}$ and thus $\mu^{\prime}=\mu$. Uniqueness for the minimizer can be established in a similar manner, as is clear. So Theorem 1 is proved.

Finally, to establish the corollary, we need only make the appropriate identifications in our payoff to ensure that $M(x, y)=-M(y, x)$.

The authors would like to thank Dr. Irving Glicksberg for his valuable comments on this paper. As a matter of fact, Dr. Glicksberg suggested an alternate proof for Theorem 1 which extends it to completely regular spaces $X, Y$. The gist of his proof involves obtaining the extended theorem by making it a corollary of Theorem 2 via a mapping: $X \rightarrow R^{n}, Y \rightarrow R^{m}$.

\section{REFERENCES}

1. M. Dresher, S. Karlin, and L. S. Shapley, Polynomial games, Ann. of Math. Study No. 24 (Princeton 1950), 161-180.

2. I. Glicksberg and 0. Gross, Notes on games over the square, Ann. of Math. Study No. 28 (Princeton 1953), 173-182.

RAND CORPORATION AND BROWN UNIVERSITY 



\section{PACIFIC JOURNAL OF MATHEMATICS}

\section{EDITORS}

\section{David Gilbarg}

Stanford University

Stanford, California

\section{R. A. Beaumont}

University of Washington

Seattle 5, Washington

\author{
A. L. Whiteman
}

University of Southern California Los Angeles 7, California

E. G. Straus

University of California

Los Angeles 24, California

\section{ASSOCIATE EDITORS}

\author{
E. F. BECKENBACH \\ C. E. BURGESS \\ M. HALL \\ E. HEWITT
}
A. HORN
V. GANAPATHY IYER
R. D. JAMES
M. S. KNEBELMAN
L. NACHBIN
I. NIVEN
T. G. OSTROM
H. L. ROYDEN

M. M. SCHIFFER

G. SZEKERES

F. WOLF

K. YOSIDA

\section{SUPPORTING INSTITUTIONS}

\author{
UNIVERSITY OF BRITISH COLUMBIA \\ CALIFORNIA INSTITUTE OF TECHNOLOGY \\ UNIVERSITY OF CALIFORNIA \\ MONTANA STATE UNIVERSITY \\ UNIVERSITY OF NEVADA \\ OREGON STATE COLLEGE \\ UNIVERSITY OF OREGON \\ OSAKA UNIVERSITY \\ UNIVERSITY OF SOUTHERN CALIFORNIA
}

\author{
STANFORD UNIVERSITY \\ UNIVERSITY OF TOKYO \\ UNIVERSITY OF UTAH \\ WASHINGTON STATE COLLEGE \\ UNIVERSITY OF WASHINGTON \\ * * * * \\ AMERICAN MATHEMATICAL SOCIETY \\ CALIFORNIA RESEARCH CORPORATION \\ HUGHES AIRCRAFT COMPANY \\ THE RAMO-WOOLDRIDGE CORPORATION
}

Mathematical papers intended for publication in the Pacific Journal of Mathematics should be typewritten (double spaced), and the author should keep a complete copy. Manuscripts may be sent to any one of the four editors. All other communications to the editors should be addressed to the managing editor, E. G. Straus at the University of California, Los Angeles 24, California.

50 reprints per author of each article are furnished free of charge; additional copies may be obtained at cost in multiples of 50 .

The Pacific Journal of Mathematics is published quarterly, in March, June, September, and December. The price per volume (4 numbers) is $\$ 12.00$; single issues, $\$ 3.50$. Back numbers are available. Special price to individual faculty members of supporting institutions and to individual members of the American Mathematical Society: $\$ 4.00$ per volume; single issues, $\$ 1.25$.

Subscriptions, orders for back numbers, and changes of address should be sent to Pacific Journal of Mathematics, 2120 Oxford Street, Berkeley 4, California.

Printed at Kokusai Bunken Insatsusha (International Academic Printing Co., Ltd.), No. 6, 2-chome, Fujimi-cho, Chiyoda-ku, Tokyo, Japan.

PUBLISHED BY PACIFIC JOURNAL OF MATHEMATICS, A NON-PROFIT CORPORATION

The Supporting Institutions listed above contribute to the cost of publication of this Journal, but they are not owners or publishers and have no responsibility for its content or policies. 


\section{Pacific Journal of Mathematics}

\section{Vol. 8, No. $4 \quad$ June, 1958}

Richard Arens, The maximal ideals of certain functions algebras ........ 641

Glen Earl Baxter, An operator identity ........................... 649

Robert James Blattner, Automorphic group representations ........... 665

Steve Jerome Bryant, Isomorphism order for Abelian groups ............ 679

Charles W. Curtis, Modules whose annihilators are direct summands...... 685

Wilbur Eugene Deskins, On the radical of a group algebra ............ 693

Jacob Feldman, Equivalence and perpendicularity of Gaussian

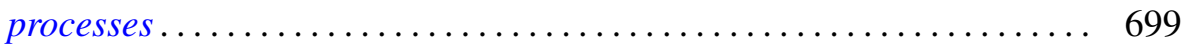

Marion K. Fort, Jr. and G. A. Hedlund, Minimal coverings of pairs by triples....................................... 709

I. S. Gál, On the theory of $(m, n)$-compact topological spaces ......... 721

David Gale and Oliver Gross, A note on polynomial and separable games........................................ 735

Frank Harary, On the number of bi-colored graphs ............... 743

Bruno Harris, Centralizers in Jordan algebras ................... 757

Martin Jurchescu, Modulus of a boundary component ............... 791

Hewitt Kenyon and A. P. Morse, Runs . . . . . . . . . . . . . . . . . . . . . . 811

Burnett C. Meyer and H. D. Sprinkle, Two nonseparable complete metric

spaces defined on $[0,1] \ldots \ldots \ldots \ldots \ldots \ldots \ldots \ldots \ldots \ldots \ldots . \ldots . \ldots . \ldots . \ldots 25$

M. S. Robertson, Cesàro partial sums of harmonic series expansions...... 829

John L. Selfridge and Ernst Gabor Straus, On the determination of numbers by their sums of a fixed order ........................ 847

Annette Sinclair, A general solution for a class of approximation

problems .................................

George Szekeres and Amnon Jakimovski, $(C, \infty)$ and $(H, \infty)$ methods of summation...................................... 867

Hale Trotter, Approximation of semi-groups of operators. ............. 887

L. E. Ward, A fixed point theorem for multi-valued functions ........... 921

Roy Edwin Wild, On the number of lattice points in $x^{t}+y^{t}=n^{t / 2} \ldots \ldots .929$ 